\title{
The effect of LLR clipping to the complexity of list sphere detector algorithms
}

\author{
Markus Myllylä, Juho Antikainen and Markku Juntti \\ Centre for Wireless Communications \\ P.O. Box 4500, FIN-90014 University of Oulu, Finland \\ \{markus.myllyla, juho.antikainen, markku.juntti\}@ee.oulu.fi
}

\author{
Joseph R. Cavallaro \\ Dept. of Electrical \& Computer Engineering \\ Rice University, Houston, TX 77251-1892, USA \\ cavallar@rice.edu
}

\begin{abstract}
The optimal detection for coded system requires the use of a maximum a posteriori (MAP) detection. A list sphere detector (LSD) can be used to approximate the MAP detector. Depending on the used list size, LSD provides a tradeoff between the performance and the computational complexity. The LSD output candidate list is used to calculate the approximation of the probability log-likelihood ratio (LLR) of each transmitted bit. The list should be large enough and it should include at least one candidate for both possible bits for good approximation. The use of a small list size causes inaccurate and, especially, very large LLRs that prevent the decoder from correcting the falsely detected signals and, thus, degrades performance. We study the effect of the LLR clipping to the performance and complexity of the LSD algorithm. We show that by limiting the dynamic range of the LLR the required LSD list size can be decreased, and, thus, the complexity of the algorithms is decreased. The optimal dynamic range values for LLR clipping are determined and the effect of the clipping to the complexity of the LSD algorithms is analyzed.
\end{abstract}

\section{INTRODUCTION}

Multiple-input multiple-output (MIMO) channels offer improved capacity and significant potential for improved reliability compared to single antenna channels [1]. MIMO techniques in combination with orthogonal frequency-division multiplexing (OFDM) (MIMO-OFDM) have been identified as a promising approach for high spectral efficiency wideband systems [2]. The bit-interleaved coded modulation (BICM) [3], [4] is a simple yet power-efficient solution to exploit the high spectral efficiency of multiple-antenna transmission.

The optimal detection for coded MIMO-OFDM system would require the use of a maximum a posteriori (MAP) detection, and the reception has to be performed separately for each subcarrier. However, the computational complexity of the optimal MAP detection is beyond the limit of most systems, and, thus, such an approach is typically not feasible. Sphere detector (SD) calculates the hard output maximum likelihood (ML) solution with reduced complexity compared to fullcomplexity ML detectors [5], [6]. However, the performance of a coded system may suffer significantly with hard ML detector compared to the optimal MAP detector. A list sphere detector (LSD) [7] is a variant of the sphere detector that can be used to approximate MAP detection.

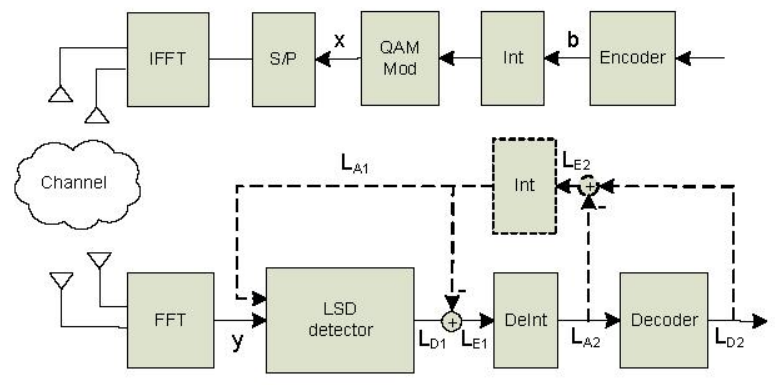

Fig. 1. A MIMO_OFDM system with $N_{T}$ transmit and $N_{R}$ receive antennas.

The LSD gives a candidate list of the transmitted symbol vectors as an output. The list can used to calculate an approximation of the probability log-likelihood ratio (LLR) of each transmitted bit. Depending on the list size, it provides a tradeoff between the performance and the computational complexity. The list should be large enough and it should include at least one candidate for both possible bits in order to obtain good approximation of the LLR. The performance of the LSD with too small a list size suffers due to inaccurate and, especially, due to very large LLRs that cause the channel decoder from correcting the falsely detected signals. In this paper, we have studied the effect of LLR clipping to the performance and complexity of the LSD algorithm. We determine the optimal dynamic range for the LLR variable and compare the performance to the system with unlimited LLR dynamic range. We show that the LLR clipping reduces the required LSD list size and, thus, the complexity of the algorithm.

The paper is organized as follows. The system model is presented in Section II. The considered LSD algorithms are presented in Section III. The log-likelihood ratio calculation and the LLR clipping are introduced in Section IV. Numerical examples are shown in Section $\mathrm{V}$ and the conclusions are drawn in Section VI.

\section{SYSTEM MODEL}

An OFDM-based multiple-antenna system with $N_{T}$ transmit antennas and $N_{R}$ receive antennas is considered with assumption $N_{R} \geq N_{T}$ and quadrature amplitude modulation constellation (QAM). The received signal can be expressed in terms of code symbol interval as

$$
\mathbf{y}_{p}=\mathbf{H}_{p} \mathbf{x}_{p}+\eta_{p}, \quad p=1,2, \ldots, P,
$$


where $P$ is the number of subcarriers, the received signal vector $\mathbf{y}_{p} \in \mathbb{C}^{N_{R} \times 1}$, the transmit symbol vector $\mathbf{x}_{p} \in \mathbb{C}^{N_{T} \times 1}$ and the noise vector $\eta_{p} \in \mathbb{C}^{N_{R} \times 1}$ are defined in the frequency domain. The elements of $\eta_{p}$ are independent and complex Gaussian with equal power real and imaginary parts, i.e., $\eta_{p} \sim$ $\mathcal{C N}\left(0, N_{0} \mathbf{I}_{N_{R}}\right)$ and represent the frequency domain thermal noise at the receiver. The channel matrix $\mathbf{H}_{p} \in \mathbb{C}^{N_{R} \times N_{T}}$ contains complex Gaussian fading coefficients with unit variance. The entries of $\mathbf{x}_{p}$ are chosen independently from a complex QAM constellation $\Omega$ with sets of $Q$ transmitted coded binary information bits $\mathbf{b}=\left[b_{1}, \ldots, b_{Q}\right]$ per symbol, i.e., $|\Omega|=2^{Q}$. The set of all possible transmitted symbol vectors is denoted by $\Omega^{N_{T}}$. The corresponding uncoded transmission rate is $R=M_{T} Q$ bits per channel use (bpcu).

A bit-interleaved coded modulation (BICM) [3], [4] is applied to the system, and the receiver consists of a soft-output detector, a deinterleaver, and a decoder. The turbo principle can be applied in the receiver so that the detector and decoder exchange the information in iterative fashion as illustrated in the block diagram of the system in Figure 1.

\section{LIST SPHERE DETECTOR}

We assume that a list sphere detector is used to provide the soft information to the decoder. The LSD algorithm gives a candidate list of the transmitted symbol vectors $\mathcal{L}$ and the corresponding Euclidean distances $d(\mathcal{L})$ as an output, which are used to calculate an approximation of the LLR. In this paper, we consider three LSD algorithms with different search strategies. The K-best-LSD algorithm [8] is a modification from K-best-LSD algorithm [9] to LSD algorithm. The SEELSD algorithm [8] is a depth-first search strategy based algorithm. The increasing radius (IR)-LSD algorithm [10] is a modification of Dijkstra's algorithm [11] to the LSD algorithm.

\section{LOG-LIKELIHOOD RATIO}

\section{A. LLR calculation}

The LLR of the $k$ th transmitted bit $b_{k}$, conditioned on the received signal vector $\mathbf{y}$, is denoted as $L_{\mathrm{D}}\left(b_{k}\right)$ and is defined to be the ratio of the conditioned probabilities of the bit taking its two possible values, i.e.,

$$
L_{\mathrm{D}}\left(b_{k}\right)=\ln \frac{P\left(b_{k}=+1 \mid \mathbf{y}\right)}{P\left(b_{k}=-1 \mid \mathbf{y}\right)} .
$$

By using the Bayes' theorem, the probability can be written as [7], [12]

$$
\begin{aligned}
L_{\mathrm{D}}\left(b_{k}\right) & =\ln \left(\frac{p\left(\mathbf{y} \mid b_{k}=+1\right)}{p\left(\mathbf{y} \mid b_{k}=-1\right)} \frac{P\left(b_{k}=+1\right)}{P\left(b_{k}=-1\right)}\right) \\
& =\ln \frac{P\left(b_{k}=+1\right)}{P\left(b_{k}=-1\right)}+\ln \frac{p\left(\mathbf{y} \mid b_{k}=+1\right)}{p\left(\mathbf{y} \mid b_{k}=-1\right)} \\
& =L_{\mathrm{A}}\left(b_{k}\right)+L_{\mathrm{E}}\left(b_{k} \mid \mathbf{y}\right),
\end{aligned}
$$

where $L_{\mathrm{A}}\left(b_{k}\right)$ is the a priori information and $L_{\mathrm{E}}\left(b_{k}\right)$ is the extrinsic information of the bits provided by the detector or decoder.
The probability of a transmitted bit $b_{k}=+1$ is equal to the sum of all the probability combinations containing a $b_{k}=+1$ for that given bit. Then, for a system containing additive white Gaussian noise (AWGN), the probability can be determined directly from the cost information known about the candidates as

$$
p\left(\mathbf{y} \mid b_{k}=+1\right)=\frac{2}{\left|\Omega_{R}\right|^{M_{T}} \sqrt{2 \pi \sigma^{2}}} \sum_{\mathbf{x} \in \Omega_{R}^{M_{T}}, b_{k}=+1} e^{\frac{-d(\mathbf{x})}{2 \sigma^{2}}},
$$

where $d(\mathbf{x})$ is the squared Euclidean distance between received vector $\mathbf{y}$ and lattice points $\mathbf{H x}$. Then the $L_{\mathrm{D}}\left(b_{k}\right)$ can be determined as

$$
\begin{aligned}
L_{\mathrm{D}}\left(b_{k}\right) & =L_{\mathrm{A}}\left(b_{k}\right)+\ln \frac{p\left(\mathbf{y} \mid b_{k}=+1\right)}{p\left(\mathbf{y} \mid b_{k}=-1\right)} \\
& =L_{\mathrm{A}}\left(b_{k}\right)+\ln \left(p\left(\mathbf{y} \mid b_{k}=+1\right)-\ln \left(p\left(\mathbf{y} \mid b_{k}=-1\right)\right),\right.
\end{aligned}
$$

where the conditional probabilities are calculated using (4). Equation (5) can then be computed using the well-known Jacobian algorithm and a small look-up table [13].

The LSD algorithm can be used to calculate an approximation of the $L_{\mathrm{D}}\left(b_{k}\right)$ by using the obtained list $\mathcal{L}$ in (4) and (5). If the size $N_{\text {cand }}$ of the list $\mathcal{L}$ is large enough, the effect of the unknown results is likely to be relatively small, and the approximation of the $L_{\mathrm{D}}\left(x_{k}\right)$ accurate enough for adequate performance. However, the performance of the LSD may suffer due to too small list size and, thus, inaccurate $L_{\mathrm{D}}\left(b_{k}\right)$ values. The error in the approximation of the $L_{\mathrm{D}}\left(b_{k}\right)$ is especially large in the case where the output list $\mathcal{L}$ of LSD includes only candidates with $b_{k}$ either +1 or -1 . This results in very large values in (5) that may cause the channel decoder not to be able to correct the falsely detected signals.

\section{B. LLR clipping}

The effect of unreliable $L_{\mathrm{D}}\left(b_{k}\right)$ may be reduced by modifying the $L_{\mathrm{D}}\left(b_{k}\right)$ values. In this paper, we study two simple methods to process $L_{\mathrm{D}}\left(b_{k}\right)$ information and the effect of the methods to the performance of a coded system. The $L_{\mathrm{D} 1}\left(b_{k}\right)$ calculated in the detector is given as $L_{\mathrm{A} 2}\left(b_{k}\right)$ input to the decoder as illustrated in Figure 1. By limiting the dynamic range of the variable, the decoder can still overcome the wrong information given as $L_{\mathrm{A} 2}\left(b_{k}\right)$ in (3).

1) Method: A very simple way to prevent very large $L_{\mathrm{D}}\left(b_{k}\right)$ values is to limit the dynamic range of $L_{\mathrm{D}}\left(b_{k}\right)$ value as [7]

$$
L_{\mathrm{D}_{\text {clip }}}\left(b_{k}\right)= \begin{cases}L_{\mathrm{D}}\left(b_{k}\right), & \text { if }\left|L_{\mathrm{D}}\left(b_{k}\right)\right| \leq L_{\mathrm{max}} \\ \operatorname{sgn}\left(L_{\mathrm{D}}\left(b_{k}\right)\right) L_{\text {max }} & \text { if }\left|L_{\mathrm{D}}\left(b_{k}\right)\right|>L_{\text {maxx }},\end{cases}
$$

where $L_{\mathrm{D}_{\text {clip }}}\left(b_{k}\right)$ is the clipped likelihood information and $L_{\text {max }}$ is the selected maximum value for $\left|L\left(b_{k}\right)\right|$.

2) Method: The other method is slightly different compared to the first one. The $L_{\mathrm{D}}\left(b_{k}\right)$ values are clipped to $L_{\max }$ if a threshold value of $L_{\text {limit }}>L_{\text {max }}$ is exceeded as

$$
L_{\mathrm{D}_{\text {clip }}}\left(b_{k}\right)= \begin{cases}L_{\mathrm{D}}\left(b_{k}\right), & \text { if }\left|L_{\mathrm{D}}\left(b_{k}\right)\right| \leq L_{\text {limit }} \\ \operatorname{sgn}\left(L_{\mathrm{D}}\left(b_{k}\right)\right) L_{\text {max }} & \text { if }\left|L_{\mathrm{D}}\left(b_{k}\right)\right|>L_{\text {limit }},\end{cases}
$$




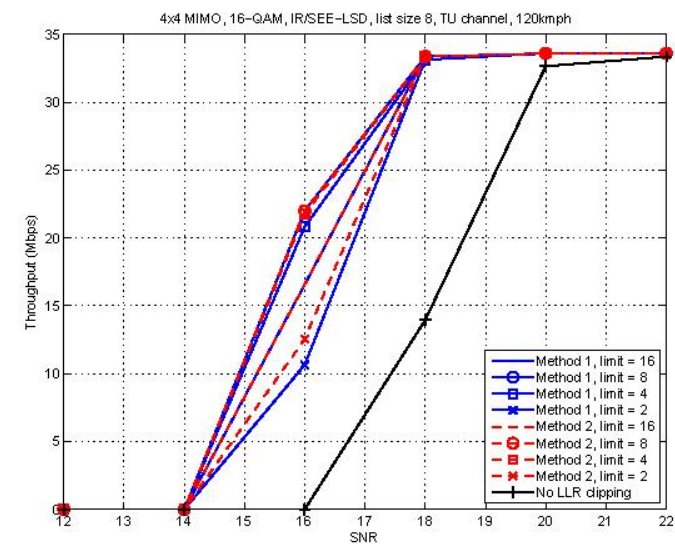

Fig. 2. Throughput vs SNR: Performance of the real IR-LSD and SEE-LSD with different LLR clipping methods and values in $4 \times 4$ antenna system with 16-QAM.

The main idea of the 2nd method is to clip only the very large $L_{\mathrm{D}}\left(b_{k}\right)$ values, which are due to small LSD list size, and bypass the $L_{\mathrm{D}}\left(b_{k}\right)$ values where both bit values are present in (5). This can be achieved by setting the $L_{\text {limit }}$ value large enough.

\section{NUMERICAL EXAMPLES}

We studied the effect of the LLR clipping to the performance of the system via computer simulations. A MIMOOFDM system was assumed with 512 subcarriers (300 used) according to 3G long term evolution (LTE) parameters [14]. A BICM with $1 / 2$ rate $[13,15]$ turbo code was applied in a typical urban (TU) 6 tap channel with a user velocity of 120 $\mathrm{kmph}$. The system was operating with $5 \mathrm{MHz}$ bandwidth at a carrier frequency of $2.4 \mathrm{GHz}$. The K-best-LSD, the SEELSD, and the IR-LSD were considered for detection and the soft outputs were decoded in an iterative turbo decoder with 8 iterations. The iterative detection and decoding was not assumed in the simulations. The K-best-LSD algorithm was applied with $C_{0}=\infty$.

\section{A. Comparison of LLR clipping methods}

We studied the effect of the two different LLR clipping methods introduced in section IV-B to the performance of the system. The simulations were executed with different $L_{\mathrm{max}}$ values to determine the optimal value to be used for clipping. The performances of the real IR-LSD/SEE-LSD with $N_{\text {cand }}=$ 8 are shown for $4 \times 416$-QAM system in Figure 2 and real K-best-LSD with $N_{\text {cand }}=64$ in Figure 3 . The method 2 is applied with $L_{\text {limit }}=100$ to clip only the very large $L_{\mathrm{D}}\left(b_{k}\right)$ values. The results show that the performance of a system is clearly improved by applying LLR clipping to limit the effect of the moderate LLR approximation.

It can be seen from Figure 2 that there is no significant performance difference between the two clipping methods with IR/SEE-LSD. However, the method 1 is clearly better with K-best-LSD in Figure 3. The reason to this is the different outputs from IR/SEE-LSD and K-best-LSD. The IR-LSD and

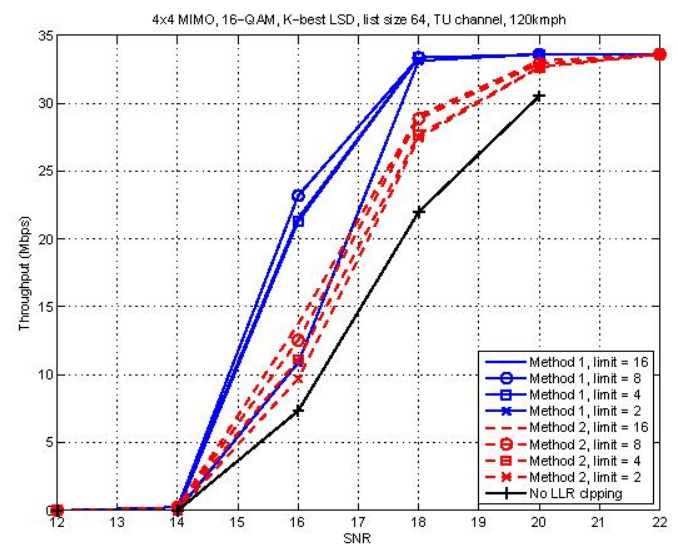

Fig. 3. Throughput vs SNR: Performance of the real K-best LSD with different LLR clipping methods and values in $4 \times 4$ antenna system with 16-QAM.

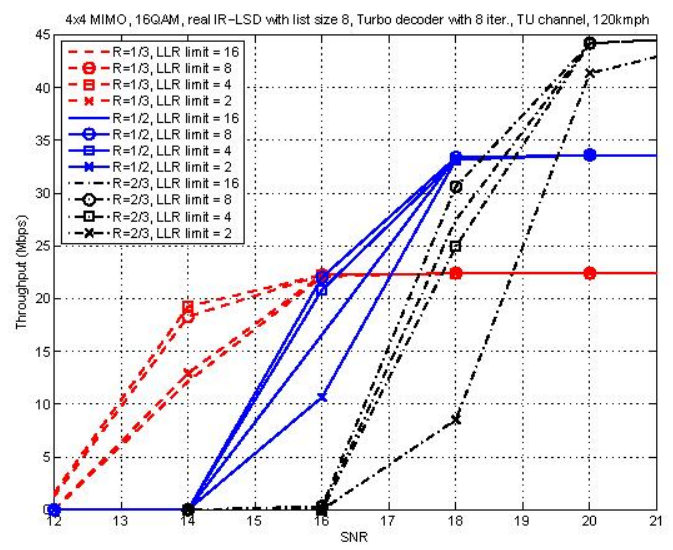

Fig. 4. Throughput vs SNR: Performance of the real IR-LSD with different code rates and LLR clipping values in $4 \times 4$ antenna system with 16 -QAM.

the SEE-LSD give the most probable candidates as an output, and, thus, the MAP approximation is rather good in the cases where both +1 and -1 are present in (5) [8], [10]. The K-bestLSD output, however, may result in a bad MAP approximation also when candidates for both bits are present in (5) [8], [10]. Thus, we conclude that the method 1 is a good choice to be applied. The simulation results show that $L_{\max }=8$ gives the best performance, which means that the dynamic range of probability $P\left(b_{k}= \pm 1 \mid \mathbf{y}\right)$ is limited between $[0.0003,0.9997]$.

We also studied if and how the code rate effects to the optimal $L_{\max }$ value at the detector. The performances of a system with code rates $1 / 3,1 / 2$, and $2 / 3$ and with different method 1 clipping values are shown in Figure 4 . We can see that with a lower code rate $1 / 3$ the values $L_{\max }=4,8$ give quite similar performance as with a higher code rate $2 / 3$ the values $L_{\max }=8,16$ show good results. The results indicate that as the decoder has more parity bits to be used in the decoding the decoder should rely less on the a priori information $L_{\mathrm{A} 2}\left(b_{k}\right)$ from the detector and the $L_{\max }$ can be set to be a lower value. However, the differences are rather small, and in practice, $L_{\max }=8$ gives good results. 


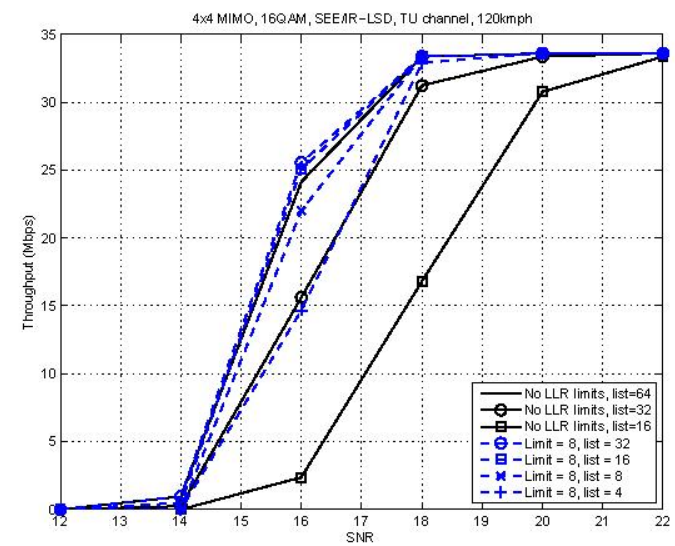

Fig. 5. Throughput vs SNR: Performance of the real IR-LSD and SEELSD with different list sizes in $4 \times 4$ antenna system with and without LLR clipping.

\section{B. Required list size}

We studied the effect of LLR clipping to the performance of a LSD based system and its effect to the required list size. The required list sizes for a system without LLR clipping have been determined in [8], [10]. The method 1 with $L_{\max }=8$ was applied for LLR clipping in the following results.

The performance of $4 \times 4$ 16-QAM system with the real IR/SEE-LSD with different list sizes is shown in Figure 5, and the same case with the real K-best-LSD with different list sizes is shown in Figure 6. It can be seen from Figure 5 that the required list size with IR/SEE-LSD decreases significantly with LLR clipping applied and in the $4 \times 4$ 16-QAM system the required list size decreases from 64 to 8 with LLR clipping. The required list size with K-best-LSD does not decrease as much as with IR/SEE-LSD as discussed earlier. However, in the same case the LLR clipping still reduces the required list size from 128 to 64 . The required list sizes were determined for $2 \times 2$ and $4 \times 4$ antenna cases with 4-QAM, 16-QAM, and 64-QAM, and the results are concluded in Table I.

The results show that the IR/SEE-LSD list size can be decreased significantly with LLR clipping. The output candidate list from the LSD includes the most probable transmitted symbol vectors, i.e., the quality of the output list is good. The performance loss without clipping is mainly due to incorrect very large LLR values which result from having erroneous $b_{k}=+1$ or $b_{k}=-1$ candidate(s) in the calculation of (2). If clipping is not introduced, the decoder receives very large incorrect $L_{\mathrm{A} 2}\left(x_{k}\right)$ from the detector and is not able to overrule it in (5).

The required list sizes of the real and complex K-best-LSD decrease with introduced LLR clipping, but the decrease is smaller than with IR/SEE-LSD. This is due to the breadth first search strategy which usually leads to having both $b_{k}=+1$ or $b_{k}=-1$ candidates in the LLR calculation, but does not provide the most probable candidates. Thus, the quality of the obtained list is not as high than with IR/SEE-LSD and a larger list size is required for similar LLR approximation.

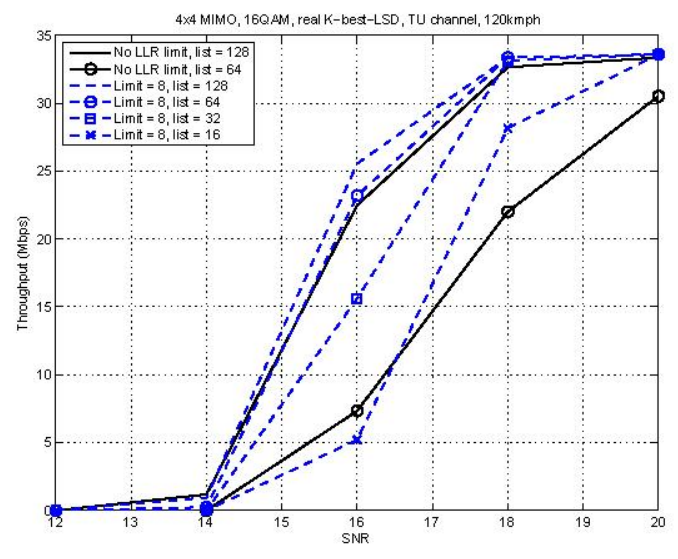

Fig. 6. Throughput vs SNR: Performance of the real K-best-LSD with different list sizes in $4 \times 4$ antenna system with and without LLR clipping.

\section{Complexity of the algorithms}

The complexity of the LSD algorithms is relative to the number of visited nodes per symbol vector [15]. The number of visited nodes by the LSD algorithm depends on the search strategy and the required list size. We studied and compared the complexity of the considered LSD algorithms with LLR clipping.

The reduced required list size with LLR clipping leads to complexity reduction in the LSD algorithm, because less number of nodes in the search tree are checked. The visited nodes by IR-LSD and SEE-LSD vary with channel realization while the K-best-LSD visits always a fixed number of nodes with radius $C_{0}=\infty$ [8], [10]. The effect of the list size to the number of visited nodes with real IR-LSD is illustrated in Figure 7. It can be seen from the figure that the average and maximum number of visited nodes decreases significantly with lower list size. In practical implementation the maximum number of nodes should be limited to fix the complexity of the algorithm [16]. The performance of the system does not degrade if the maximum limit is set high enough.

The maximum node limits were determined via computer simulations for different antenna and modulation cases. The determined values for real IR-LSD and real SEE-LSD are shown in Table II, and the determined values for real and complex K-best-LSD are shown in Table III. The ratio of the determined values with LLR clipping and without clipping is shown in brackets for the cases which were studied. It can be seen from the results that LLR clipping in general reduces the number of required checked nodes in the detector significantly. The real IR-LSD, real SEE-LSD, and real K-

TABLE I

LIST SIZES FOR IR/SEE-LSD*, REAL K-BEST-LSD ${ }^{\dagger}$ AND COMPLEX K-BEST-LSD ${ }^{+}$WITH LLR CLIPPING

\begin{tabular}{|c|c|c|}
\hline & $2 \times 2$ & $4 \times 4$ \\
\hline 4-QAM & not studied & $N_{\text {cand }}=8^{*} / 32^{\dagger} / 32^{\ddagger}$ \\
16-QAM & $N_{\text {cand }}=8^{*} / 16^{\dagger} / 16^{\ddagger}$ & $N_{\text {cand }}=8^{*} / 64^{\dagger} / 128^{\ddagger}$ \\
64-QAM & $N_{\text {cand }}=16^{*} / 64^{\dagger} / 64^{\ddagger}$ & $N_{\text {cand }}=16^{*} / 128^{\dagger} / 256^{\ddagger}$ \\
\hline
\end{tabular}




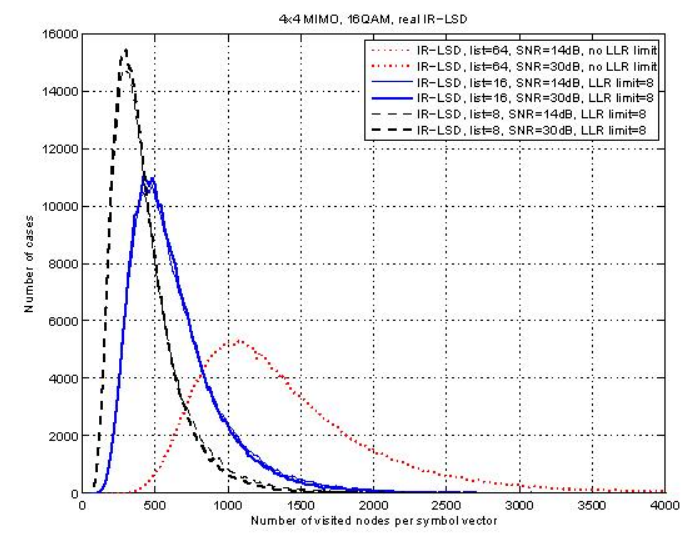

Fig. 7. Histogram of the number of visited nodes per symbol vector by the real $\mathbb{R}$-LSD algorithm in TU channel with $4 \times 4$ 16-QAM system.

best-LSD complexity is reduced in most of the cases when as the complexity of the complex K-best-LSD is only reduced with $4 \times 4$ 64-QAM configuration. It should also be noted that the real SEE-LSD does not perform satisfactory without LLR clipping and search node limiting [16], but with LLR clipping feasible limits can be determined for all the cases except $4 \times 4$ 64-QAM. The number of visited nodes gives a guideline in comparison of the complexity of different algorithms, but the exact complexity difference is dependent on the applied architecture and implementation.

\section{CONCLUSIONS}

The effect of the LLR clipping to the performance and complexity of the LSD algorithm was studied. We compared two different clipping methods with different clipping values, and the method 1 with $L_{\max }=8$ was shown to be a good and simple choice to be used. We also noticed that the applied code rate has effect on the optimal $L_{\max }$ value. However, in practice the effect is small and $L_{\max }=8$ value can be used. We showed that by limiting the dynamic range of the LLR the required LSD list size can be decreased in many cases, and, thus, the complexity of the algorithms is decreased. The complexity reduction of the LSD algorithms was studied, and feasible maximum limits for search were defined for IR-LSD

TABLE II

DETERMINED MAXIMUM NODE LIMITS FOR REAL IR-LSD ${ }^{\dagger}$ AND REAL SEE-LSD $\ddagger$ WITH LLR CLIPPING

\begin{tabular}{|c|c|c|}
\hline & $2 \times 2$ & $4 \times 4$ \\
\hline 4-QAM & not studied & $250^{\dagger} / 300^{\ddagger}$ \\
16-QAM & $80^{\dagger}(53 \%) / 150^{\ddagger}(50 \%)$ & $800^{\dagger}(27 \%) / 3500^{\ddagger}$ \\
64-QAM & $200^{\dagger}(33 \%) / 400^{\dagger}$ & $2000^{\dagger}(50 \%) />14000^{\ddagger}$ \\
\hline
\end{tabular}

TABLE III

NUMBER OF VISITED NODES WITH REAL K-BEST-LSD ${ }^{\dagger}$ AND COMPLEX K-BEST-LSD ${ }^{\ddagger}$ WITH LLR CLIPPING

\begin{tabular}{|c|c|c|}
\hline & $2 \times 2$ & $4 \times 4$ \\
\hline 4-QAM & not studied & $254^{\dagger}(100 \%) / 212^{\ddagger}(100 \%)$ \\
16-QAM & $148^{\dagger}(44 \%) / 272^{\ddagger}(100 \%)$ & $1364^{\dagger}(57 \%) / 4368^{\ddagger}(100 \%)$ \\
64-QAM & $1096^{\dagger}(68 \%) / 4160^{\ddagger}(100 \%)$ & $5704^{\dagger}(27 \%) / 36928^{\ddagger}(53 \%)$ \\
\hline
\end{tabular}

and SEE-LSD.

\section{ACKNOWLEDGEMENTS}

This work was done in MITSE project which was supported by Elektrobit, Nokia TP, Nokia-Siemens Networks, Texas Instruments and the Finnish Funding Agency for Technology and Innovation, Tekes. The first author would like to thank Mr. Jari Ylioinas for helpful comments and fruitful discussions.

\section{REFERENCES}

[1] A. Goldsmith, S. Jafar, N. Jindal, and S. Vishwanath, "Capacity limits of MIMO channels," IEEE Joumal on Selected Areas in Communications, vol. 21, no. 5, pp. 684-702, June, 2003.

[2] H. Yang, "A road to future broadband wireless access: MIMO-OFDMbased air interface," Communications Magazine, IEEE, vol. 43, no. 1, pp. 53-60, Jan., 2005.

[3] G. Caire, G. Taricco, and E. Biglieri, "Bit-interleaved coded modulation," IEEE Trans. Inform. Theory, vol. 44, no. 3, pp. 927 - 946, May 1998.

[4] S. H. Muller-Weinfurtner, "Coding approaches for multiple antenna transmission in fast fading and ofdm," IEEE Trans. Signal Processing, vol. 50 , no. 10 , pp. $2442-2450$, Oct. 2002 .

[5] U. Fincke and M. Pohst, "Improved methods for calculating vectors of short length in a lattice, including a complexity analysis," Math. Comput., vol. 44, no. 5, pp. 463-471, May 1985.

[6] M. O. Damen, H. E. Gamal, and G. Caire, "On maximum-likelihood detection and the search for the closest lattice point," IEEE Trans. Inform. Theory, vol. 49, no. 10, pp. 2389-2402, Oct. 2003.

[7] B. Hochwald and S. ten Brink, "Achieving near-capacity on a multipleantenna channel," IEEE Trans. Commun, vol. 51, no. 3, Mar. 2003.

[8] M. Myllylä, P. Silvola, J. Cavallaro, and M. Juntti, "Comparison of Two Novel List Sphere Detector Algorithms for MIMO-OFDM Systems," in Proc. IEEE Int. Symp. Pers., Indoor, Mobile Radio Commun. (PIMRC), Helsinki, Finland, Sep 11 - 14, 2006.

[9] K.Wong, C. Tsui, R.-K. Cheng, and W. Mow, "A VLSI architecture of a K-best lattice decoding algorithm for MIMO channels," in Proc. IEEE ISCAS'02, vol. 3, Helsinki, Finland, Jun. 11-14 2002, pp. 273-276.

[10] M. Myllylä, J. Cavallaro, and M. Juntti, "A List Sphere Detector based on Dijkstra's Algorithm for MIMO-OFDM Systems," in Proc. IEEE Int. Symp. Pers., Indoor, Mobile Radio Commun. (PIMRC), Athens, Greece, Sep 12 - 19, 2007.

[11] E. W. Dijkstra, "A note on two problems in connexion with graphs," in Numerische Mathematik, vol. 1, Mathematisch Centrum, Amsterdam, Netherlands, 1959, pp. 269-271.

[12] J. Hagunauer, E. Offer, and L. Papke, "Iterative Decoding of Binary Block and Convolutional Codes," IEEE Trans. Inform. Theory, vol. 42, no. 2, Mar. 1996.

[13] P. Robertson, E. Villebrun, and P. Hoeher, "A comparison of optimal and sub-optimal MAP decoding algorithms operating in the log domain," Proc. IEEE Int. Conf. Commun. (ICC), pp. 1009-1013, 1995.

[14] 3rd Generation Partnership Project (3GPP), "TSGR1\#41 R1-050-520, EUTRA downlink numerology," 3rd Generation Partnership Project (3GPP), Tech. Rep., 2005.

[15] A. Burg, M. Borgmann, M. Wenk, M. Zellweger, W. Fichtner, and H. Bölcskei, "VLSI Implementation of MIMO Detection Using the Sphere Decoding Algorithm," IEEE Joumal of Solid-State Circuits, vol. 40 , no. 7 , Jul. 2005 .

[16] M. Myllylä, M. Juntti, and J. Cavallaro, "Implementation Aspects of List Sphere Detector Algorithms," in Proc. IEEE Global Telecommun. Conf. (GLOBECOM), Washington, D.C., USA, Nov 26 - 30, 2007. 